\title{
Assessment of Factors Influencing Lymph Node Count in Colorectal Cancer
}

\author{
Suleyman Altintas 1 and Mehmet Bayrak² \\ 1Departmentof Pathology, Adana City Hospital, Adana, Turkey \\ ${ }^{2}$ Department of General Surgery, Adana Ortadogu Private Hospital, Adana, Turkey
}

\begin{abstract}
Objective: To evaluate the factors that influence pericolorectal lymph node (LN) retrieval from colectomy specimens in colorectal in cancer staging.

Study Design: Descriptive study

Place and Duration of Study: Ortadogu Private Hospital Adana, Turkey, between January 2012 and March 2018.

Methodology: The inclusion criteria was right hemi-colon, left hemi-colon and rectum carcinoma specimen, respectively. The exclusion criteria was total colectomies due to synchronous colorectal carcinomas and cancers developed on the basis of ulcerative colitis and familial polyposis coli. Age and gender of the patients, the length of the colectomy specimen, the location and size of the tumor-node-metastasis (TNM) stage, and the count of retrieved lymph nodes were evaluated.

Results: Among the 145 patients in this study, the mean count of recovered and metastatic lymph nodes was 32.5 and 2.9 , respectively. Sufficient lymph node assessment was done in 130 of the 145 patients. The number of lymph node yields was independently associated with depth of invasion, T-classification, tumor size, AJCC/UICC stage as well as right-sided tumor location. The count of harvested lymph nodes significantly decreased in patients who received preoperative therapy. Increased node counts were associated with increased overall survival (OS).

Conclusion: Tumor site and size, length of the surgical specimen, T-classification, depth of invasion and AJCC/UICC stage are predictors for the lymph node numbers in colorectal cancer surgery. Retrieval of removed nodes and counting helps in staging and number of removed nodes, and affects survival.
\end{abstract}

Key Words: Pericolorectal lymph nodes, Retrieval colorectal carcinoma, TNM classification, Neoadjuvant therapy, Understaging.

How to cite this article: Altintas S, Bayrak M. Assessment of factors influencing lymph node count in colorectal cancer. J Coll Physicians Surg Pak 2019; 29(12):1173-1178.

\section{INTRODUCTION}

Colorectal carcinoma is one of the most commonly diagnosed cancers, globally. Retrieval of pericolorectal lymph nodes from colectomy specimens is important in colorectal cancer staging. Several researches have detected that the increased count of nodes is associated with an increased disease-free and overall survival (OS). ${ }^{1}$ Obtaining more lymph nodes allows for more accurate cancer staging and, therefore, is important in planning further therapies, especially adjuvant chemotherapy.

However, the optimal count of lymph nodes that needs to be assessed is controversial. Some major series show that it should be needed at least 12-14 nodes to get adequate prognostic information. ${ }^{2}$ The International Union Against Cancer, the American Joint Cancer Committee, and the National Cancer Institute consensus panel have suggested that minimum 12 nodes be

Correspondence to: Suleyman Altintas, Departmentof Pathology,

Adana City Hospital, Adana, Turkey

E-mail: suleymanveysmn@hotmail.com

Received: December 06, 2018; Revised: October 23, 2019;

Accepted: October 24, 2019 evaluated to ensure adequate sampling. 3 However, more and more evidence suggests that this rod indicates that the probability of finding metastasis increases as the number of nodes examined increases, which suggests that a minimum number of nodes can stage all patients not correctly or reliably. ${ }^{4}$ Population-based data indicate that only $37 \%$ of colon cancer patients have sufficient LN assessment. 5

The count of $L N$ taken can be influenced not only by surgeons and pathologists, but also by factors independent of surgeons and pathologists. There are many factors influencing the final node count.

The aim of this study was to evaluate the factors that influence pericolorectal $\mathrm{LN}$ retrieval from colectomy specimens in colorectal cancer staging.

\section{METHODOLOGY}

Between January 2012 and March 2018, 145 patients with colorectal cancer underwent radical surgery at Ortadogu Private Hospital in Adana, Turkey. Study design was a descriptive research, and it was approved by the University Hospital Clinical Ethics Committee. Open surgery was performed in all the patients by single 
surgeon; and depending upon the site of the tumor, a conventional colonic resection with $\mathrm{LN}$ excision was conducted. Preoperative neoadjuvant chemoradiotherapy (CRT) was given in 29 patients in primary rectal cancer.

Following 24-hour initial fixation in buffered formal saline, the mesentery in the excised sample was dissected from the intestine. LNs were then obtained visually or by palpation through the whole mesentery and pericolorectal areas. Two or three hematoxylin and eosin stained sections were incised from each LN block. All the specimens were examined by the same pathologist.

The inclusion criteria was right hemi-colon, left-hemi colon and rectum carcinoma specimen. The exclusion criteria was total colectomies due to synchronous colorectal carcinomas and cancers developed on the basis of ulcerative colitis and familial polyposis coli. After excluding total colectomies, a total of 145 cases were involved in this work. The gender and age of the patients, the length of the specimen, the location and size of the tumors, the depth of invasion, the number of retrieved and metastatic LNs were studied after the surgery. The depth of invasion and the count of metastatic LNs supported stage of the disease using the 7th edition of the AJCC/UICC TNM classification. 6 The site of the tumors was categorised into the right colon (from the cecum to the distal transverse colon), the left colon (from the splenic flexure to the sigmoid colon) and the rectum.

Statistical analysis was carried out using SPSS package programme (Version 22.0, SPSS Inc., Chicago, IL, USA). The normality for each continuous variable was controlled by Kolmogorov-Smirnov and Shapiro-Wilk tests and histogrammes. All numerical data were expressed as median values (minimum-maximum) or proportions.

Intergroup comparisons were normally compared with Student's t test for distributed data or One-Way ANOVA, Mann-Whitney U-test for normally non-distributed data, or Kruscall-Wallis test. OS is the interval from the time of diagnosis to death from any cause. OS was analysed using the Wald test, and when different parameters were applied, the log-rank test was used to examine their association. The survival curve was plotted using the standard Kaplan-Meier methodology. $P<0.05$ was considered significant.

Cox regression model was used to define the variables. Then, the therapy effect set for these selected variables was calculated. Cox model was also used to investigate the interaction of treatment effect with subgroup status in a discovery analysis. Differences in the best overall response rates between the treatment groups were analysed with the Cochran-Mantel-Haenszel test. Receiver operating characteristic curves (ROC curves) were constructed and the areas under curve (AUC) as well as sensitivity and specificity were calculated. $\mathrm{P}<0.05$ was considered significant.

\section{RESULTS}

Table I summarises the overall features of the 145 patients with colorectal cancer. Ninety-two (63.4\%) were men and $53(36.6 \%)$ were women, ranging in age from 22 to 88 years (median age $=58$ years).

The metastatic and total count of LNs associated with gender, tumor location, age, specimen length, size of the tumour, diagnostic histology, depth of invasion, $\mathrm{T}$ and $\mathrm{N}$ classification is shown in Table II.

The mean count of recovered and metastatic lymph nodes was 32.5 and 2.9 respectively. Sufficient LN assessment (i.e. evaluation of at least 12 LNs) was carried out in 130 of the 145 (89.7\%) patients. Eleven $(7.6 \%)$ of the patients with inadequate evaluation were the rectal cancer patients who received neoadjuvant therapy.

The count of LNs recovered from the patients younger than 50 years was higher than that from the elderly age groups; however, it was not statistically significant $(p=0.155)$. There was a slight but not statistically significant difference between men and women.

Importantly, more LNs were retrieved from cancers in both the right colon and left colon than from rectum. The mean specimen length was $22.5 \pm 16.3 \mathrm{~cm}$. The longer the colorectal specimen, the higher were the numbers of recovered LNs $(p=0.0001)$. Increased tumor size

Table I: General characteristics of the study population.

\begin{tabular}{lc}
\hline Characteristics & No. of Patients (\%) \\
\hline Sex & $92(63.4 \%)$ \\
Men & $53(36.6 \%)$ \\
Women & \\
\hline Diagnostic histology & $28(19.3 \%)$ \\
Well differentiated adenocarcinoma? & $99(68.3 \%)$ \\
Moderately differentiated adenocarcinoma? & $7(4.8 \%)$ \\
Poorly differentiated adenocarcinoma? & $11(7.6 \%)$ \\
Mucinous carcinoma & $12(8.3 \%)$ \\
\hline Pathological tumor classification & $127(87.6 \%)$ \\
cT2 & $6(4.1 \%)$ \\
cT3 & \\
cT4 & $67(46.2 \%)$ \\
Clinical or pathological lymph node classification & $41(28.3 \%)$ \\
N0 & $37(25.5 \%)$ \\
N1 & $118(81.4 \%)$ \\
N2 & $10(6.9 \%)$ \\
\hline Stage & $29(20 \%)$ \\
2 & \\
3 & \\
Received preop neoadjuvant therapy & \\
Alatus & \\
Expired & \\
\hline
\end{tabular}


Table II: The metastatic and total number of lymph nodes associated with different clinicopathological parameters.

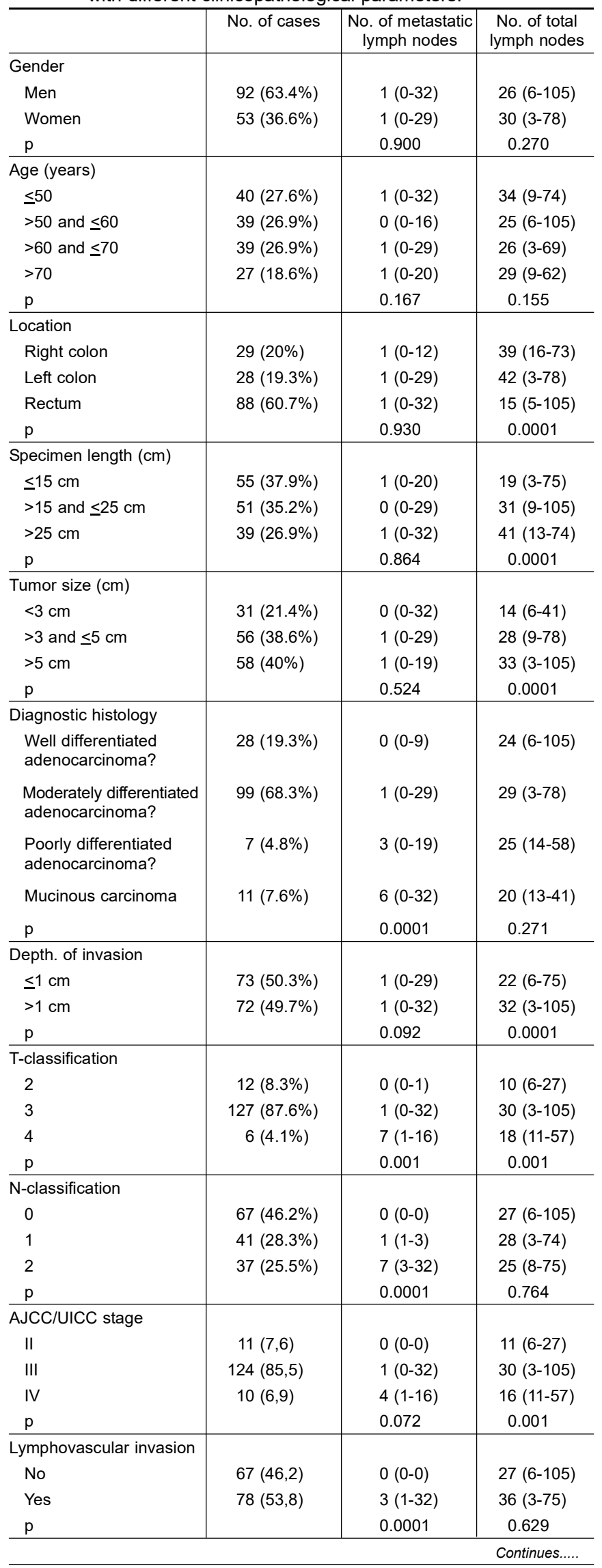

\begin{tabular}{l|c|c|c} 
& No. of cases & $\begin{array}{c}\text { No. of metastatic } \\
\text { ymmph nodes }\end{array}$ & $\begin{array}{c}\text { No. of total } \\
\text { lymph nodes }\end{array}$ \\
\hline Perineural invasion & $121(83,4)$ & $0(0-20)$ & $29(6-105)$ \\
No & $24(16,6)$ & $8(0-32)$ & $23(3-71)$ \\
Yes & & 0.0001 & 0.242 \\
\hline$p$ & $29(20 \%)$ & $0(0-14)$ & $14(6-22)$ \\
\hline Preop neoadjuvant therapy & $116(80 \%)$ & $1(0-32)$ & $32(3-105)$ \\
Yes & & 0.124 & 0.0001 \\
No & & & \\
p & $118(81.4 \%)$ & $0(0-20)$ & $28(6-105)$ \\
Status & $27(18.6 \%)$ & $2(0-32)$ & $22(3-62)$ \\
Alive & & 0.001 & 0.034 \\
Ex & &
\end{tabular}

positively related with higher count of LNs $(p=0.0001)$. Although more metastatic LNs were detected as the grade increased, there was no association with the total count of recovered $\mathrm{LNs}(\mathrm{p}=0.802)$.

The total count of LNs increased significantly with depth of invasion. $(p=0.0001)$. There was a definite association between both the metastatic and total count of LNs and advancing $T$ stage $(p=0.001)$. The total number of recovered LNs did not significantly change according to nodal status. Metastatic LNs were higher in the presence of lymphovascular and perineural invasion, but the total count of harvested LNs was not significantly affected.

Twenty-nine (20\%) patients with rectal carcinoma had presurgical treatment with 11 of them having less than 12 LNs examined. The count of retrieved LNs importantly decreased in patients who received preoperative therapy $(p=0.0001)$.

During follow-up, 27 (18.6\%) patients died. Estimated OS of the patients was $59.1 \pm 2.2$ months. One-year survival ratio was $89 \%(n=129)$, and 5 year-survival ratio was $77 \%(n=112)$ (Figure 1).

Cox regression analysis were carried out to determine the factors affecting survival. OS decreased when the age, length of the specimen and the count of metastatic $\mathrm{LNs}$ increased. $\mathrm{LN}$ yields proved to be an independent prognostic factor for OS of the patients. ROC analysis showed that the cut-off value should be more than 20 (sensitivity $72.9 \%$, specificity $48.1 \%$ and accuracy $63 \%$ ) (95\% Cl 0.52-0.74) (Figure 2).

\section{DISCUSSION}

In colorectal carcinoma, metastasis to regional LNs is a significant prognostic factor and is used to select the most appropriate cancer treatment. ${ }^{3}$ Patients with nodenegative disease have a 5 -year survival rate of $70-80 \%$, conversely it is $30-60 \%$ in patients with node-positive disease. Adjuvant chemotherapy improves survival in the latter group. Recurrence between $20 \%$ and $30 \%$ in obviously completely excised tumors without lymph node metastases is thought to be due to occult LN 
disease. If this subgroup can be identified by better LN staging, they can also benefit from adjuvant chemotherapy. ${ }^{7}$

In this work, $89.7 \%$ of patients had sufficient LN assessment, which is importantly higher than that of mean value in the literature. It is important to understand the factors that affect $L N$ involvement to identify and maximise diagnostic information from LNs. Many factors have been revealed in the literature to influence the LN yields: the patient (age, BMI, individual differences), the specimen type, tumour factors, previous treatment modalities, skills of surgeon and pathologist. But it is hard to determine, which is important.2,5,8

Patient factors like older age is associated with decreased LN recovery in many studies. ${ }^{5}$ However, in this study, although there was a slight decrease in older

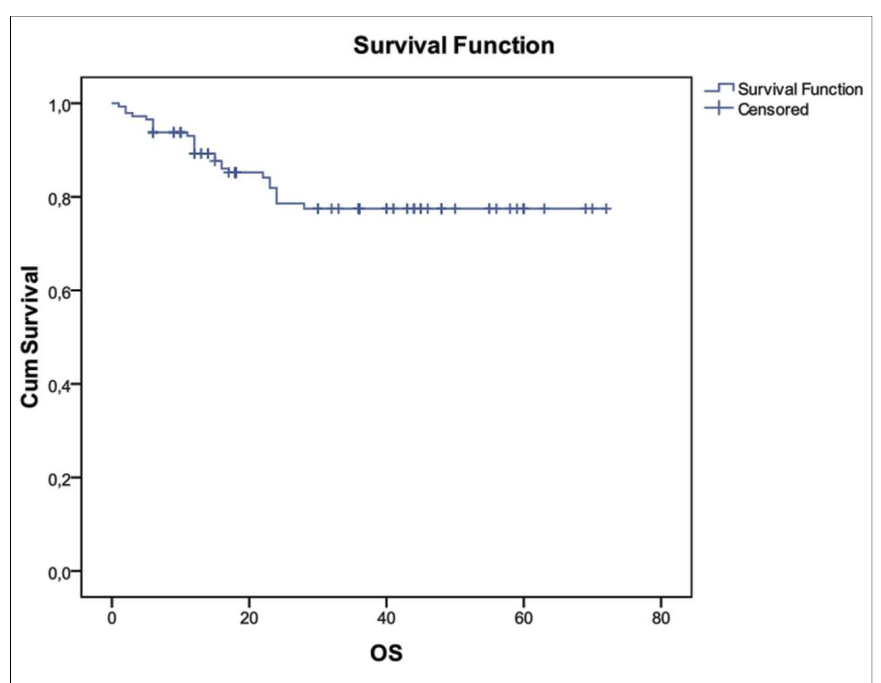

Figure 1: Kaplan-Meier survival curve of 5-year overall survival of the patients.

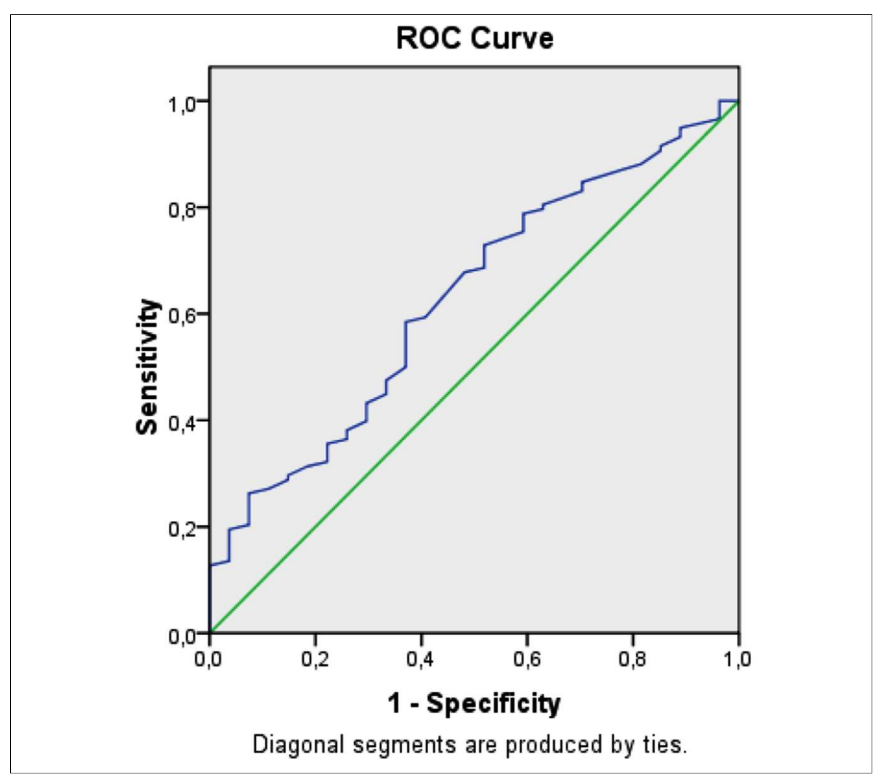

Figure 2: ROC curve analysis. age group, there was no significant correlation with increasing age. Gender seems to have no effect in many studies; but some mentioned greater sampling in females. ${ }^{9,10}$ In this research, the total count of retrieved LNs in women was slightly higher than men, but the difference was not statistically substantial.

One finding of this study was that larger tumors were more probably the result in a higher nodal yield - a result which was consistent with many previous studies. ${ }^{11,12}$ No biological explanation has yet been provided for this finding, but larger tumors are likely to cause greater inflammatory responses in the mesenteric basin, which may lead to more LNs. ${ }^{13}$

This data also showed that the depth of wall invasion in the colon was clearly related to the count of recovered LNs. Greater intestinal penetration may provide larger antigenic inflammatory and immune responses within regional LNs, making them more susceptible to pathological examination. ${ }^{5}$ In this study, there was a positive correlation between total $\mathrm{LN}$ number and progressive T-stage and higher tumor stage. In a population-based study, Kelder et al. also showed that the T-stage was independently correlated with the number of LNs studied. ${ }^{14}$ In that research, the number of LNs recovered increased with advanced TNM stage, but Tsai et al. found controversial results in their study. ${ }^{13}$ According to some studies, a higher number of LNs were retrieved in patients with $\mathrm{LN}$ involvement by tumor ${ }^{11}$; however, this study did not show this association. In a research by Nash et al., 15 there was no association between the total count of lymph nodes examined and the number of LNs with metastatic disease.

Many researches have conducted a relationship between tumor differentiation subtype and LN recovery, whereas the poorly differentiated tumor is associated with greater LN harvest as compared to well differentiated lesion. ${ }^{15}$ In this study, although more metastatic LNs were found in less differentiated tumours; tumor differentiation did not significantly affect the total count of LNs.

Another determinant factor for lymph node harvest is tumor location. Generally, the right side of the colon is considered to be correlated with a greater count of LNs with respect to the left side of the colon and rectum. ${ }^{5}$ This study also found the same results. One explanation for this difference was that during the right colectomy, larger mesenteric lymphatic fragments can be excised according to the left colectomy sequence. ${ }^{16}$ Another explanation is that surgical samples obtained from rightsided colon excisions were found to be longer than leftsided excisions. ${ }^{12}$ Many studies, such as this research, have shown the relationship between the length of the colorectal sample and the lymph node acquisition.9,10,12 The longer the colorectal sample, the higher the count of LNs taken. 
Many rectal cancer patients receive preoperative radiotherapy, with or without chemotherapy to reduce the number of metastatic LNs and to shrink the size of the tumor. This neoadjuvant treatment has been shown to cause significant reduction in both size and count of lymph nodes present in the post-resection examination.5,17 Several independent studies, investigating the number of LNs taken after long-term CRT, showed a continuous decrease in the number of LNs compared to nonirradiated samples. The average number of nodes detected varies from 4 to 14 per sample. 5,17 In this study, an approximate mean of 14 odes was retrieved per sample. A significantly lower total node count was obtained in patients with neoadjuvant therapy than those without preoperative treatment. This study supports the assumption that preoperative long-term CRT reduces the number of cumulative LNs in the colorectal sample. ${ }^{18}$ Preoperative radiation (with or without chemotherapy) reduces the number of nodes examined, possibly due to LN atrophy, fibrosis and lymphocyte depletion. 5,19

In this study, both the higher metastatic and total node counts correlated with improved OS. The count of LNs involved may reflect a patient's improved immune response; as such, the association between node counts and survival may be confounded by the tumorhost response, since a stronger immunologic response leads to improved survival. 20 Le Voyer et al. showed that for colon cancer patients with positive LNs, there was an absolute improvement in 5-year OS, if more LNs were analysed. ${ }^{21}$ Chen et al. also showed that the median survival in colon cancer, when 1-7, 8-14 and >15 LNs were examined, was 46,52 and 67 months respectively $(p$ <0.001).22 In this study, ROC analysis showed that the cut-off value of LNs retrieved should be more than 20.

Although all the surgeries were done by the same surgeon in this study, surgeon-dependent factors must also be taken into account in LN counts. Certainly, the extent of resection is determined by the surgeon in the operating room. The role of the surgeon is to excise the involved segment of colon with en bloc lymphadenectomy of the drainage $L N s$ associated with proximal ligation of the open veins and the nourishing vascular pedicle. ${ }^{23}$ If an incomplete excision is undertaken, this can account for 'under-staging' with inadequate surgical $\mathrm{LN}$ yield.

Beyond all of these, many studies suggest that tissue handling by pathologists may be an important factor in LN harvest from colon cancer samples. ${ }^{8}$ Increasingly, evidence indicates that as the greater the number of nodes examined, the greater the likelihood that metastasis will be found, suggesting that no minimum number of nodes accurately or reliably stages all patients. ${ }^{24,25}$ In this study, at least one metastatic LN could be found in more than half of the patients. In plenty of pathology laboratories, macroscopic evaluation and colorectal cancer dissection of samples are sometimes transferred to trainee pathologists who have little experience and expertise. Even in skilled hands, the finding of LNs by palpation in the mesenteric fat requires pain and a lot of time. In the light of these findings, a pathologist should try to obtain the maximum count of LNs in any sample; at least, to decrease the pathologist factor.

Limitation of this research includes its retrospective nature, thus all the variables should be assessed in prospective and multicentric studies.

\section{CONCLUSION}

Results found in this study indicate that tumor location and size, length of the operation specimen, T-classification, depth of invasion, and higher AJCC/UICC stage are affecting factors for the LN counts in colorectal cancer operations, independent of pathologist and surgeon factors. Standard neoadjuvant therapy significantly reduces the count of LNs after proctectomy in patients with rectal cancer. In colorectal cancer, inadequate LN recovery may lead to understaging and inadequate adjuvant therapy, which may contribute to recurrence of disease and shortened cancer-specific survival.

According to this data, the increasing number of nodes is significantly associated with OS, so at least, 20 LNs are needed, although minimum $12 \mathrm{LNs}$ are recommended in both latest surgical and histopathological practices.

\section{ETHICAL APPROVAL:}

Cukurova University Clinical Ethical Board approved this study; and ethical approvals were obtained prior to initiation of the research work.

\section{PATIENTS' CONSENT:}

Informed consents were obtained from the patients to publish the data concerning this case.

\section{CONFLICT OF INTEREST:}

Authors declared no conflict of interest.

\section{AUTHORS' CONTRIBUTION:}

SA: Conducted immunohistological and pathological examinations and interpreted the patients' data regarding advanced stage colorectal disease.

MB: Performed the operations and interpreted the patients' data regarding advanced stage colorectal disease.

Both authors read and approved the final manuscript.

\section{REFERENCES}

1. Chang GJ, Rodriguez-Bigas MA, Skibber JM. Lymph node evaluation and survival after curative resection of colon cancer: systematic review. J Natl Cancer Inst 2007; 99:433-41.

2. Igali L. Lymph node count in colorectal cancer - Do we have the final answer? J Gastrointest Oncol. 2012; 3:301-3. 
3. Nelson H, Petrelli N, Carlin A. Guidelines 2000 for colon and rectal cancer surgery. J Natl Cancer Inst 2001; 93:583-96.

4. Greenson JK, Bonner JD, Ben-Yzhak O. Phenotype of microsatellite unstable colorectal carcinomas. Am J Surg Pathol 2003; 27:563-70.

5. Baxter NN, Virnig DJ, Rothenberger DA. Lymph node evaluation in colorectal cancer patients: A population-based study. J Natl Cancer Inst.2005; 97:219-25.

6 International Union Against Cancer (2009). TNM classification of malignant tumours, 7th edn. Wiley-Blackwell, West-Sussex.

7. Ong M, Schofield JB. Assessment of lymph node involvement in colorectal cancer. World J Gastrointest Surg 2016; 8:179-92.

8. Tonini V, Birindelli A, Bianchini S, Cervellera M, Bacchi Reggiani ML, Wheeler J, et al. Factors affecting the number of lymph nodes retrieved after colo-rectal cancer surgery: A prospective single-centre study. Surgeon 2019; 30065-4.

9. Norwood MG, Sutton AJ, West K, Sharpe DP, Hemingway D, Kelly MJ. Lymph node retrieval in colorectal cancer resection specimens: National standards are achievable, and low numbers are associated with reduced survival. Colorectal Dis 2010; 12:304-9.

10. Fan L, Levy M, Aguilar CE, Mertens RB, Dhall D, Frishberg DP, et al. Lymph node retrieval from colorectal resection specimens for adenocarcinoma: Is it worth the extra effort to find at least 12 nodes? Colorectal Dis 2011; 13:1377-83.

11. Onitilo AA, Stankowski RV, Engel JM, Doi SAR. Adequate lymph node recovery improves survival in colorectal cancer patients. J Surg Oncol 2013;107:828-34.

12. Yang L, Xiong Z, Xie Q, He W, Liu S, Kong P, et al. Prognostic value of total number of lymph nodes retrieved differs between left-sided colon cancer and right-sided colon cancer in stage III patients with colon cancer. BMC Cancer 2018; 18:558.

13. Tsai HL, Huang CW, Yeh YS, Ma CJ, Chen CW, Lu CY, et al. Factors affecting number of lymph nodes harvested and the impact of examining a minimum of 12 lymph nodes in stage IIII colorectal cancer patients: A retrospective single institution cohort study of 1167 consecutive patients. BMC Surg 2016; 16:17.

14. Kelder W, Inberg B, Schaapveld M, Karrenbeld A, Grond J, Wiggers $\mathrm{T}$, et al. Impact of the number of histologically examined lymph nodes on prognosis in colon cancer: A population-based study in the Netherlands. Dis Colon Rectum 2009; 52:260-7.
15. Nash GM, Row D, Weiss A, Shia J, Guillem JG, Paty PB, et al. A predictive model for lymph node yield in colon cancer resection specimens. Ann Surg 2011; 253:318-22.

16. Prandi M, Lionetto R, Bini A, Francioni G, Accarpio G, Anfossi A, et al. Prognostic evaluation of stage $B$ colon cancer patients is improved by an adequate lymphadenectomy: Results of a secondary analysis of a large scale adjuvant trial. Ann Surg 2002; 235:458-63.

17. Wichmann MW, Müller C, Meyer G, Strauss T, Hornung HM, Lau-Werner U, et al. Effect of preoperative radiochemotherapy on lymph node retrieval after resection of rectal cancer. Arch Surg 2002; 137:206-10.

18. Tekkis PP, Smith JJ, Heriot AG, Darzi AW, Thompson MR, Stamatakis JD. A national study on lymph node retrieval in resectional surgery for colorectal cancer. Dis Colon Rectum 2006; 49:1673-83.

19. Marks JH, Valsdottir EB, Rather AA, Nweze IC, Newman DA, Chernick MR. Fewer than 12 lymph nodes can be expected in a surgical specimen after high-dose chemoradiation therapy for rectal cancer. Dis Colon Rectum. 2010; 53:1023-9.

20. Pages F, Berger A, Camus M. Effect or memory T cells, early metastasis, and survival in colorectal cancer. $N$ Engl $J$ Med 2005; 353:2654-66.

21. Le Voyer TE, Sigurdson ER, Hanlon AL, Mayer RJ, Macdonald JS, Catalano PJ, et al. Colon cancer survival is associated with increasing number of lymph nodes analyzed: A secondary survey of intergroup trial INT-0089. J Clin Oncol 2003; 21: 2912-9.

22. Chen SL, Bilchik AJ. More extensive nodal dissection improves survival for stages I to III of colon cancer: A population-based study. Ann Surg.2006; 244:602-10.

23. Fleshman J, Sargent DJ, Green E. Laparoscopic colectomy for cancer is not inferior to open surgery based on 5-year data from the COST study group trial. Ann Surg 2007; 246:655-62.

24. Birbeck KF, Macklin CP, Tiffin NJ. Rates of circumferential resection margin involvement vary between surgeons and predict outcomes in rectal cancer surgery. Ann Surg 2002; 235:449-57.

25. Washington MK, Berlin J, Branton P, Burgart LJ, Carter DK, Fitzgibbons $\mathrm{PL}$, et al. Protocol for the examination of specimens from patients with primary carcinoma of the colon and rectum. Arch Pathol Lab Med 2009; 133:1539-51. 